\title{
Tumor pardo parasinfisiario en paciente con enfermedad renal crónica terminal: reporte de caso y revisión de la literatura
}

\section{Wilson A. Delgado Azañero' José Leonardo Silva Toro ${ }^{2}$ Edgar Alejandro Cabrera Gómez²}

Profesor Investigador Extraordinario. Residente del Programa de Especialización en Cirugía Oral y Maxilofacial.

Facultad de Estomatología. Universidad Peruana Cayetano Heredia.

\section{Correspondencia}

Wilson A. Delgado Azañero

Av. Honorio Delgado 430 - Lima 31, Perú

Teléfono: (511) 381-1950 (252)

e-mail: wilson.delgado@upch.pe

Recibido : 18 de setiembre de 2011

Aceptado : 10 de noviembre de 201

Delgado-Azañero WA, Silva-Toro JL, Cabrera-Gómez EA. Tumor pardo parasinfisiario en paciente con enfermedad renal crónica terminal: reporte de caso y revisión de la literatura. Rev Estomatol Herediana. 2011; 21(4):219-225.

\section{RESUMEN}

Se reporta y discuten las características de un tumor óseo, ubicado en la zona parasinfisiaria derecha en una paciente adolescente de 15 años de edad, con diagnóstico sistémico de enfermedad renal crónica terminal (ERCT). La lesión ósea mandibular, estudiada por imaginología 3D, correlacionada con los hallazgos clínicos, la histopatología del tejido obtenido por punción aspiración y los datos de la historia médica, establecieron el diagnóstico de tumor pardo en hiperparatiroisdismo secundario causado por la ERCT. Adicionalmente, se encontró pérdida de la lámina dura de los dientes y alteraciones del trabeculado óseo de los maxilares, falanges y cráneo. Se enfatiza la importancia de la punción- aspiración como procedimiento confiable, fácil y libre de complicaciones para el diagnóstico histopatológico de tumor pardo.

Palabras clave: HIPERPARATIROIDISMO / GRANULOMA DE CÉLULAS GIGANTES / INSUFICIENCIA RENAL CRÓNICA.

Brown tumor of parasymphysis in a patient with end-stage renal disease: case report and literature review

ABSTRACT

The features of an osseous tumor located in the right part of the chin of a 15-years-old girl patient suffering of end-stage renal disease (ESRD) is reported and discussed. The jaw lesion studied by 3D imaginology, correlated with the clinical findings, histopathology of tissue obtained by aspiration puncture and data registered in the medical history of the patient established the diagnosis of brown tumor in secondary hyperparathyroidism caused by ESRD. In addition, lost of lamina dura of teeth and alterations of bone trabecula of mandible and maxilla, phalanges and cranium was identified. It is emphasize the importance of the aspiration puncture as trusted, easy and free of complication procedure to make the diagnosis of brown tumor.

Key words: HYPERPARATHYROIDISM / GIANT CELL GRANULOMA / CHRONIC RENAI INSUFFICIENCY.

\section{Introducción}

El tumor pardo es una lesión no neoplásica de los huesos, que aparece como resultado del aumento de los niveles circulantes de paratohormona debido a severo hiperparatiroidismo, donde el metabolismo óseo es anormal y donde existe activación de la reabsorción ósea osteoclástica, primariamente en el hueso cortical (1-3).

El hiperparatiroidismo se clasifica en tres categorías: primario, secundario y terciario. También se reconoce un cuadro muy raro denominado hiperparatiroidismo ectópico, en el cual el incremento de la hormona paratiroidea no depende del hiperfuncionamiento de la glándula paratiroides sino de tumores malignos del ovario, riñón, pulmón o de un tumor neuroectodérmico primitivo, los cuales pueden producir
PTH intacta y proteínas similares dando lugar a un cuadro clínico semejante al hiperparatiroidismo primario $(4,5)$.

El hiperparatiroidismo primario (HPTP) se caracteriza por hipercalcemia, el cual es resultado de los niveles altos de paratohormona; del 80 al 85\% de casos se debe a un adenoma solitario, el $10 \%$ a hiperplasia de todas las glándulas, $4 \%$ a dos o más adenomas y menos del $1 \%$ puede ocurrir por carcinoma de paratiroides (6). Para el diagnóstico de estas alteraciones es fundamental realizar estudios de ultrasonido del cuello y gammagrafía con Tc99msestamibi (7).

La prevalencia del HPTP se ha estimado en $1 \%$ en la población adulta, con una incidencia de 1/100,000 personas por año, es más común en mujeres que en hombres, es muy raro en niños y adolescentes y es más frecuente a partir de la quinta década de vida (8).

Los síntomas y signos asociados a hipercalcemia varían con la edad, el grado y duración del cuadro. Los ancianos son más sensibles a la presencia de niveles altos de calcemia. La hipercalcemia afecta a todos los órganos, pero con particular importancia al sistema nervioso central, riñón y huesos. Las anormalidades esqueléticas en el HPTP ocurren entre el 1,4 a $14 \%$ de los casos. Puede haber desmineralización ósea en el $30 \%$ de los casos con distribución variable entre huesos corticales (huesos largos) y huesos trabeculares (vértebras). Los huesos maxilares también son afectados.

La lesión clásica del tumor pardo es la denominada osteítis fibrosa quística, en donde existe reabsor- 
ción ósea con sustitución del tejido óseo por tejido fibroso y células gigantes multinucleadas (osteoclastos) dando lugar a cambios radiográficos parecen quistes. Adicionalmente se pueden observar diversos grados de desmineralización ósea, como puede ser disminución de la densidad del trabeculado y esfumación de la lámina dura que rodea las raíces de los dientes (9).

El hiperparatiroidismo secundario (HPTS) es una respuesta fisiológica a hipocalcemia, es decir, aparece cuando existe una menor disponibilidad de calcio iónico como ocurre en enfermos con insuficiencia renal crónica, malabsorción de calcio, osteomalacia, en la deficiencia de vitamina D (raquitismo), en el pseudohiperparatiroidismo, $\mathrm{y}$ cuando existe pérdida renal crónica de calcio como es en los casos de hipercalciuria idiopática. Se diferencia del HPTP en que en éste, el crecimiento de las paratiroides es autónomo y quizás irreversible, mientras en el secundario se produce una hiperplasia adaptativa y quizás reversible en todas las glándulas paratiroides (10).

La insuficiencia renal crónica es la causa más frecuente de HPTS. En el 90\% de pacientes con insuficiencia renal crónica se diagnostica este cuadro cuando inician la hemodiálisis. La combinación de hiperfosfatemia y la disminución de la producción renal de 1,25-dihidroxivitamina $\mathrm{D}$ dan lugar a la menor disponibilidad de calcio, que al persistir produce hiperplasia de las células principales de las glándulas paratiroides con el consecuente incremento de la secreción de paratohormona.

En el HPTS de observa una progresiva alteración de los huesos, dando lugar a cuadros de osteítis fibrosa quística, similar a los que se observan en el HPTP. También ocurren calcificaciones a nivel de los tejidos blandos y cardiovasculares. En conjunto estas alteraciones se denominan osteodistrofia renal (11).

Al igual que en el HPTP, en los huesos se desarrollan tumores pardos, los cuales, en algunos casos, se desarrollan a nivel de los huesos maxilares manifestándose como deformaciones asintomáticas de las tablas óseas vestibulares, también se observa desaparición de la lámina dura de los dientes y rarefacciones óseas por disminución del trabeculado (12-14). La mayoría de pacientes con HPTS son tratados medicamente con calcio y vitamina D y la administración de quelantes de fosfato en el intestino para disminuir la hiperfosfatemia. Un pequeño porcentaje de pacientes (1 a $2 \%)$ requieren paratiroidectomía cuando no responden al tratamiento médico.

El hiperparatiroidismo terciario, fue descrito por primera vez en 1963, se considera la forma más rara de hiperparatiroidismo. En esta entidad se produce una secreción autónoma de PTH en pacientes con hiperparatiroidismo secundario de larga evolución. Este fenómeno se sebe a una marcada estimulación y conversión de las glándulas paratiroides de un estado de hiperplasia reversible (policlonal) a un defecto de crecimiento irreversible (monoclonal) con marcada hiperfunción glandular, que lleva a la formación de adenomas autónomos que segregan elevadas cantidades de PTH independiente de las fluctuaciones de la concentración de calcio sanguíneo, semejando el cuadro clínico de HPTP.

Se estima que el 50\% de pacientes con trasplante renal desarrollan HPT terciario y se cree que está relacionado con un mayor tiempo de duración de la diálisis antes del trasplante. En el hiperparatiroidismo terciario, existe una proliferación autónoma de la glándula paratiroides con resistencia a la terapia supresora con calcio y vitamina D, fenómeno que se atribuye a inactivación monoclonal de un factor supresor del crecimiento, localizado en el cromosoma 11 (15).

Los cambios a nivel esquelético son similares a los observados en las dos formas anteriores señaladas de HPT. En relación con el tratamiento se ha reportado que la paratiroidectomía total o parcial, con autotrasplante de tejido paratiroideo normal corrige los trastornos óseos y metabólicos, mejorando los síntomas y la calidad de vida de los pacientes (16).

El diagnóstico de cualquier categoría de HPT se establece sobre la base de los hallazgos clínicos, de laboratorio y estudios imaginológicos. Es fundamental determinar los niveles de calcio sérico y/iónico, fósforo sérico, de PTH y calcio en orina de 24 horas.

Los estudios por imágenes permiten identificar las alteraciones óseas, como son, desmineralización ósea generalizada, reabsorción subperióstica de las falanges distales y del tercio externo de la clavícula, tumores pardos y pérdida de la lámina dura de los dientes entre4 otras alteraciones. En el cráneo aparecen rarefacciones óseas características, denominadas de sal y pimienta.

Para la localización de adenomas se usa con frecuencia el ultrasonido y la gammagrafía con Tecnecio 99-sestamibi. La Tomografía Computarizada y la Resonancia Magnética son indispensables para determinar si se trata de una enfermedad multiglandular (hiperplasia o multiadenomas), así como para 
descartar la presencia de glándulas paratiroides ectópicas.

En este reporte se describe un caso de tumor pardo de la zona parasinfisiaria en una adolescente con insuficiencia renal crónica que se encuentra recibiendo diálisis por largo tiempo. El diagnóstico se realizó por punción aspiración del tumor óseo.

\section{Reporte de Caso}

En agosto de 2011, el Servicio de Nefrología del Hospital Nacional Cayetano Heredia, solicitó al Servicio de Cirugía Oral y Maxilofacial de la Facultad de Estomatología de la Universidad Peruana Cayetano Heredia, la evaluación de una paciente femenina de 15 años de edad con un tumor en la zona mentoniana, padecía de enfermedad crónica terminal, recibía diálisis durante varios años y estaba en espera para un trasplante renal.

La paciente presentaba marcada asimetría facial debida a una lesión de aspecto tumoral ubicada en la zona parasinfisiaria derecha, de varios meses de evolución, crecimiento lento, de consistencia dura y que no ocasionaba ninguna sintomatología (Fig. 1,2).

En el examen intraoral se encontró, en el maxilar superior, desaparición del surco vestibular debido a la presencia de abultamientos asimétricos ubicados a nivel de los incisivos y caninos, siendo más pronunciado en la zona del incisivo lateral y canino izquierdo. En la mandíbula, el surco vestibular estaba conservado, pero se notaba una deformación en la zona más inferior a nivel de las piezas 43 y 45 , cerca a la tabla basal (Fig. 3). La palpación de las deformaciones intraorales de ambos maxilares mostraba, en general, una consistencia dura, pero en la lesión del mentón se detectaban focos de consistencia blanda. Ninguna de las zonas afectadas producía sintomatología.

Estudio imaginológico: Se tomó radiografía panorámica, tomografías tipo cone beam de los maxilares y radiografías de manos y cráneo, que permitieron identificar las siguientes alteraciones:

En la radiografía panorámica: alteración ósea caracterizada por disminución de la densidad del trabeculado de los maxilares y desaparición de la lámina dura de todos dientes, presencia de una lesión osteolítica parcialmente corticalizada, ubicada en la zona parasinfisiaria que se extendía desde la raíz de la pieza 43 hasta la parte más distal de la raíz de la pieza 46, comprometía la tabla ósea basal produciendo su

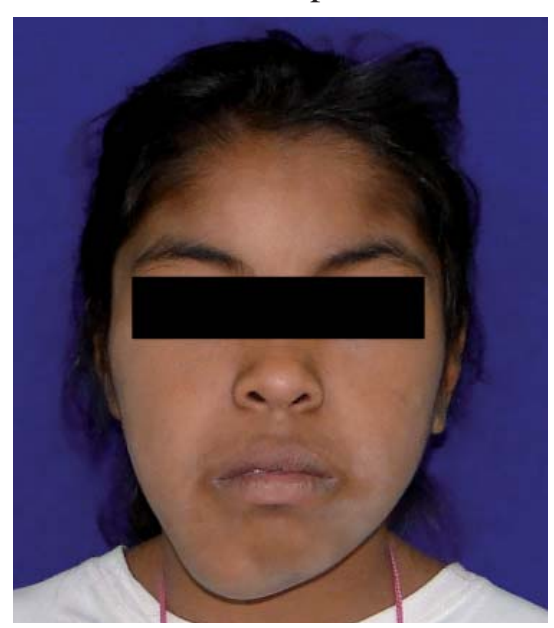

Fig. 1. Marcada asimetría facial por deformación de la zona parasinfisiaria derecha.

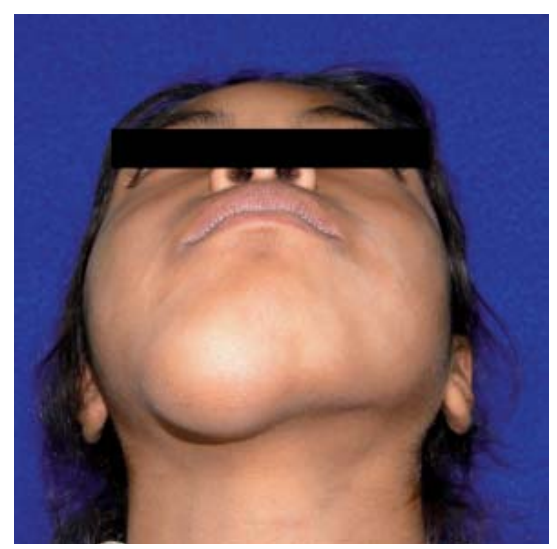

Fig. 2. La deformación compromete el borde inferior de la tabla mandibular. adelgazamiento; dentro de la lesión radiolúcida se observaban líneas radiopacas delgadas distribuidas irregularmente, en parte formando imagen de panal de abejas (Fig. 4).En cortes panorámicos de radiografías tipo cone beam se podía apreciar mejor el marcado adelgazamiento de la tabla ósea basal y la tabicación interna (Fig. 5).

En cortes coronales se observaba una imagen radiolúcida expansiva con una cortical externa muy delgada con fina tabicación interior que producían una imagen multilocular (Fig.6 y 7). Del mismo modo, este tipo de imagen se podía apreciar en los cortes sagitales (Fig. 8).En la reconstrucción 3D de la mandíbula se observa el desplazamiento y adelgazamiento de la cortical basal y la compartimentación interna de la lesión parasinfisiaria (Fig. 9).

En la radiografía de la mano derecha, se identificó una imagen radiolúcida ubicada en el extremo proximal de la falange del cuarto dedo que producía una ligera expansión ósea (Fig. 10). La radiografía frontal del cráneo, mostraba alteraciones del trabeculado óseo de la bóveda caracterizadas por adelgazamiento marcado de la cortical e imagen de sal y pimienta a nivel del díploe (Fig.11).

Punción aspiración de la lesión: Con la finalidad de establecer el diagnóstico definitivo de la lesión mandibular, se procedió a realizar

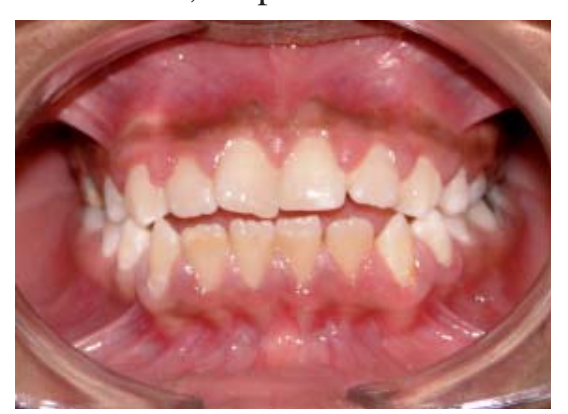

Fig. 3. Desaparición del surco vestibular antero-superior por deformación ósea de la zona. 

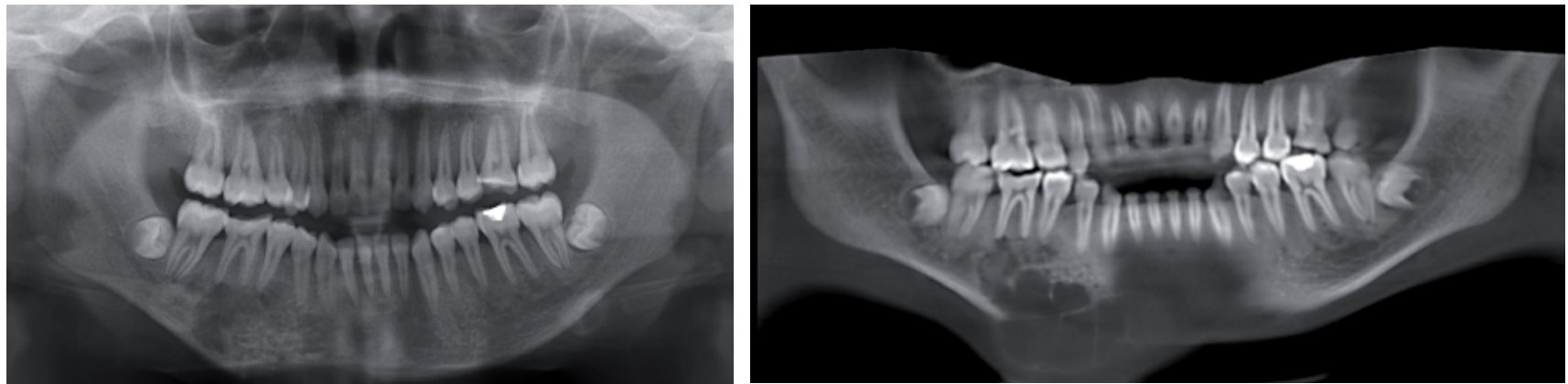

Fig. 4. Radiografía panorámica donde se observa: pérdida de la Fig. 5. Radiografía panorámica tipo cone beam donde se distingue deslámina dura de los dientes, alteraciones del trabeculado óseo en plazamiento y marcado adelgazamiento de la tabla ósea basal y tabicación ambos maxilares, imagen radiolúcida redondeada con tabicación interna de la lesión. interna con patrón de panal de abejas ubicada en la zona para sinfisiaria derecha.

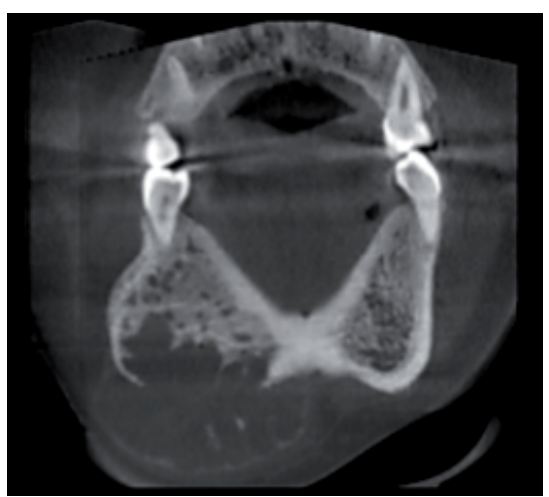

Fig. 6. Tomografía, corte coronario a nivel de premolar donde se observa imagen radiolúcidas expansiva, con fina cortical y tabicación interna.

un punción aspiración, obteniéndose fragmentos de un tejido blando con áreas sanguinolentas, que en conjunto se podía describir como un material blando hemorrágico de color pardo (Fig. 12), el cual fue fijado en formol y sometido al proceso de rutina para obtener cortes en parafina para estudio histopatológico.

Estudio histopatológico: los cortes teñidos con H\&E mostraban células gigantes multinucleadas dentro de un estroma fibroso, adyacentes al cual existían zonas hemorrágicas, estableciéndose el diagnóstico de granuloma central de células gigantes (Fig.13).

Otros estudios: En la historia clínica de la paciente se hallaron los siguientes resultados de análisis de sangre: hematocrito $27 \%$; hemoglobina 8,35g/dL; eritrocitos 2970000/ $\mathrm{mm}^{3}$; leucocitos $6430 / \mathrm{mm}^{3}$; plaque-

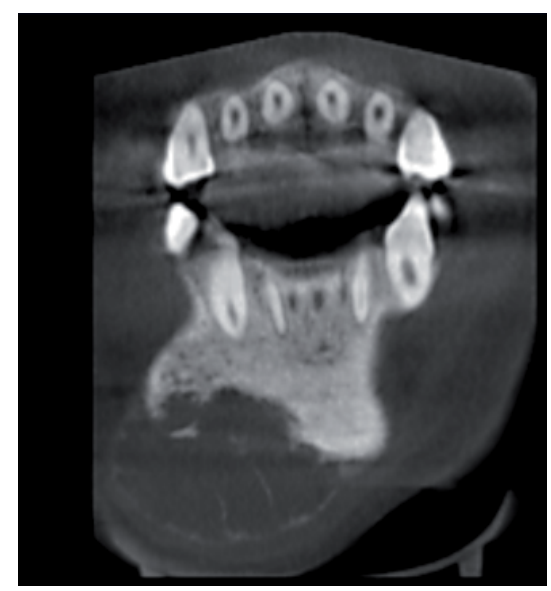

Fig. 7. Tomografía, corte coronario a nivel de canino donde se observa imagen radiolúcida expansivas, con fina cortical y tabicación interna.

tas 247000 / $\mathrm{mm}^{3}$; calcio $9,1 \mathrm{mg} / \mathrm{dl}$; dosaje de paratohormona 620pg/ml, fosfatasa alcalina $649 \mathrm{U} / \mathrm{L}$.

Diagnóstico de la lesión: La correlación de los hallazgos clínicos, imaginológicos, diagnóstico histopatológico de granuloma central de células gigantes, incremento notable de la cantidad paratohormona y fosfatasa alcalina en sangre y el cuadro sistémico de enfermedad renal crónica terminal, llevaron a la conclusión que la lesión ósea mandibular correspondía a un tumor pardo desarrollado por un cuadro de hiperparatiroidismo secundario, asociado a la condición renal de la paciente.

Con el diagnóstico señalado, el Servicio de Nefrología del Hospital Nacional Cayetano Heredia inició

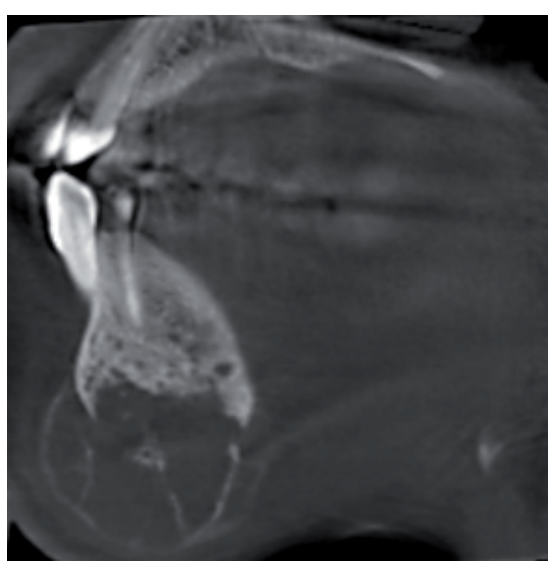

Fig. 8. Tomografía, corte sagital a nivel de canino donde observa las características de la lesión expansiva como en las figuras 6 y 7.

la terapia medicamentosa con Calcitriol EV 1mg tres veces por semana y por vía oral con dosis de $0,25 \mathrm{mg}$ en dos tomas al día. La paciente continúa en fase de diálisis, en preparación para un trasplante renal.

\section{Discusión}

La enfermedad renal crónica terminal, con frecuencia conduce al desarrollo de un amplio síndrome clínico relacionado con alteraciones del metabolismo óseo y mineral y otros cambios dependientes de la función renal, como son: anormalidades del calcio, fósforo, paratohormona, del metabolismo de la vitamina $\mathrm{D}$, anormalidades de la renovación ósea; de la mineralización, volumen, crecimiento lineal y resistencia del hueso y calcificaciones a nivel del sistema cardiovascular y de tejidos blandos (17). 


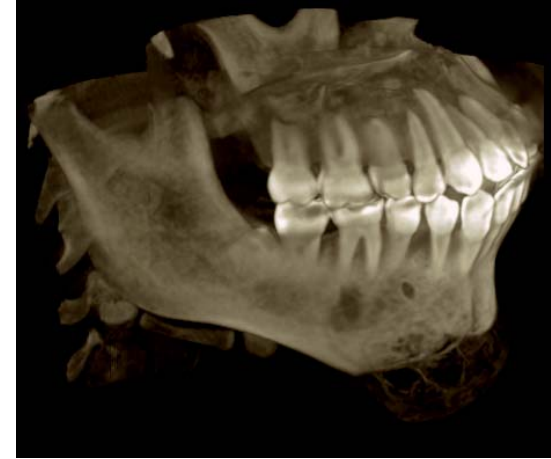

Fig. 9. Reconstrucción 3D de la lesión mandibular: distensión y adelgazamiento de la tabla basal y fina tabicación interna.

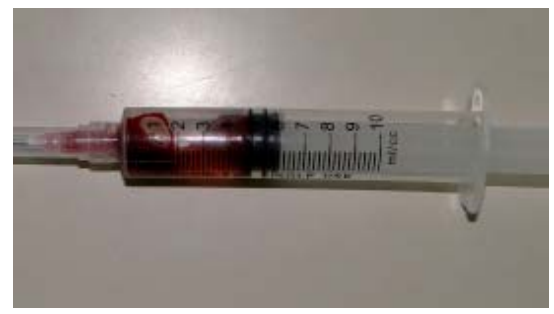

Fig. 12. Material sanguinolento obtenido por punción aspiración de la lesión mandibular.

El término osteodistrofia renal solía utilizarse para referirse a las alteraciones señaladas, pero actualmente está exclusivamente reservado para los cambios óseos, comprobados por patología, que son debidos a hiperparatiroidismo secundario y deficiencia de calcitriol (18) .

En los pacientes con ERCT se han descrito cambios radiográficos asintomáticos a nivel de los maxilares, caracterizados por pérdida de la lámina dura alveolar y alteraciones del trabeculado óseo (19), pero la presencia de lesiones expansivas no se consideraba un hallazgo frecuente. A partir de año 1966, se han descrito casos de hiperparatiroidismo secundario en los cuales existía deformación y expansión de las corticales de los maxilares, descritos como tumores localizados en la mandíbula, maxilar superior, paladar o en algunos casos, semejando cuadros de macrognatia (20-29).

Según Lerman et al. (30), hasta la fecha se han reportado en la lite-

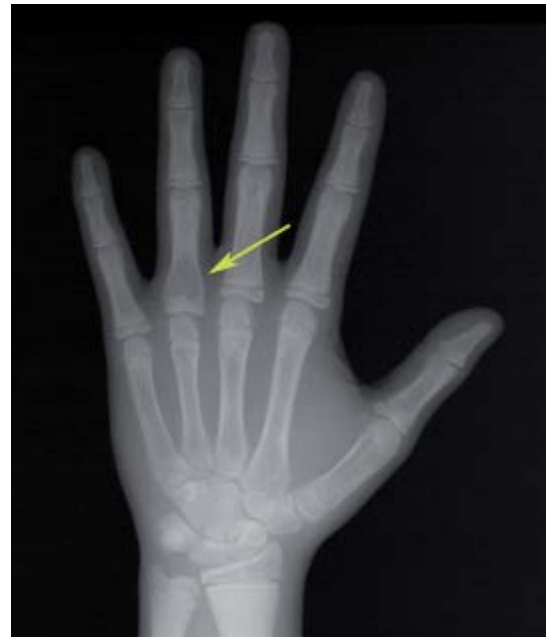

Fig. 10. Radiografía de mano derecha conde se observa una imagen radiolúcida, ligeramente expansiva ubicada en el extremo proximal de la falange de cuarto dedo (flecha).

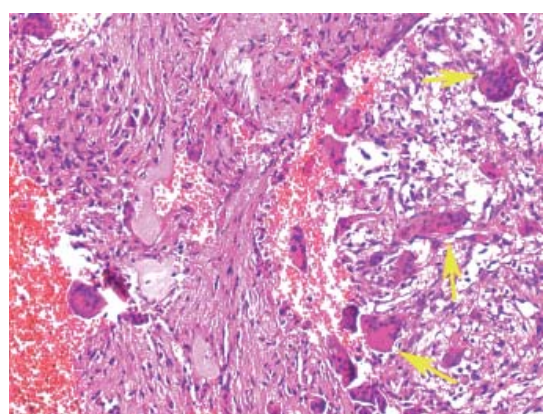

Fig. 13. RMicrofotografía del tejido aspirado: células gigantes multinucleadas (flechas), zonas hemorrágicas y estroma fibroso. H\&E 200X.

ratura en inglés 23 casos de expansión de los maxilares en cuadros de enfermedad renal crónica con desorden del metabolismo óseo y mineral. La mayoría de casos se presentaron como agrandamientos difusos de la mandíbula o de la maxila y no como crecimientos localizados.

Los autores mencionados (30) han reportado dos casos de tumores expansivos localizados en la mandíbula que se desarrollaron en dos pacientes que padecían de enfermedad renal crónica terminal, uno de los pacientes era un varón de 33 años con un tumor localizado en el tercer cuadrante y el otro era una paciente de 23 años con un tumor duro ubicado entre las piezas 34 y 34 . Al

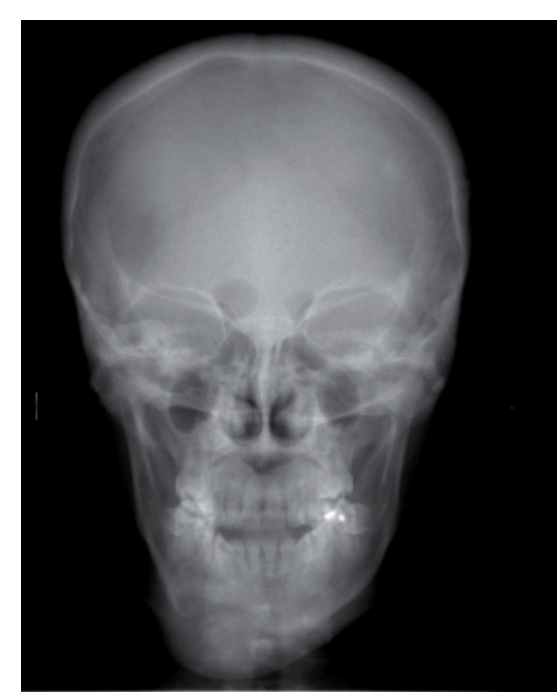

Fig. 11. Radiografía de cráneo donde se observa imagen de sal y pimienta en el díploe y alteración de las corticales.

igual que nuestro caso eran lesione óseas localizadas y expansivas, por consiguiente este tipo de alteraciones óseas se pueden presentarse en la evolución de los pacientes con diagnóstico de enfermedad renal crónica terminal y probablemente sean más frecuentes de lo que se reconoce en la literatura.

Si bien en el caso que reportamos, la historia médica de la paciente, el cuadro clínico y los cambios óseos identificados por imaginología respaldaban fuertemente el diagnóstico de osteítis fibrosa quística asociado a HPTS, es necesario comentar que la lesión, desde el punto de vista clínico y radiográfico, podía corresponder a una lesión fibro-ósea benigna, a un quiste o a un tumor odontogénico. Por otro lado, la pérdida de la de la lámina dura de los dientes y las alteraciones del trabeculado óseo de los maxilares también pueden observarse en la displasia fibrosa y en otras enfermedades más raras de nuestro medio como es la enfermedad de Paget. Este raciocinio resalta la importancia de haber establecido el diagnóstico de granuloma central de células gigantes (tumor pardo) 
de nuestro caso, mediante estudio histopatológico.

El diagnóstico definitivo de granuloma central de células gigantes usualmente se realiza mediante una biopsia de la lesión, en nuestro caso, el diagnóstico histológico se pudo establecer por un procedimiento simple de punción aspiración, el cual consistió en, previa anestesia infiltrativa de la zona, obtener fragmentos de tejido de la lesión utilizando una jeringa Luer con una aguja No. 18.

El material obtenido en la punción aspiración fue fijado en formol neutro al $10 \%$ y luego sometido al proceso de rutina para obtener láminas histológicas teñidas con H\&E. Es importante enfatizar que en lesiones óseas donde se presume la presencia de tejido blando, el estudio histológico de células en bloque, como se denomina al procedimiento llevado a cabo en nuestro caso, es altamente confiable y preciso. Como ventajas adicionales de la punción aspiración se puede señalar que es un método simple, rápido, indoloro y no requiere la utilización de sutura.

Los tumores pardos son lesiones óseas caracterizadas por la presencia de células gigantes multinucleadas dentro de un estroma fibroso con extensas zonas hemorrágicas y depósitos de hemosiderina. Fueron descritas inicialmente en hiperparatiroidismo primario, pero, como se ha mencionado también se presentan en hiperparatiroidismo secundario (31). Los tumores pardos son el resultado de la destrucción ósea por osteoclastos, los que han aumentado en su población y actividad como consecuencia del incremento de la paratohormona. Radiograficamente se presentan como lesiones radiolúcidas corticalizadas uni o multiloculares.
En el caso que reportamos se diagnostico un tumor pardo en la zona parasinfisiaria derecha y se identificaron alteraciones radiográficas del trabeculado óseo en ambos maxilares, esfumación de la lámina dura de los dientes, una lesión radiolúcida ligeramente expansiva en el extremo proximal de la falange del cuarto dedo de la mano derecha e imágenes de sal y pimienta nivel de la calota craneal (Figs. 4-11). Todas estas alteraciones del tejido óseo correspondían a un cuadro de hiperparatiroidismo secundario, que desarrolló la paciente en la evolución de la ERCT que padecía.

En la enfermedad renal crónica terminal no se producen niveles adecuados de vitamina $\mathrm{D}$ por parte del riñón, lo que ocasiona una disminución de la absorción de calcio por el intestino que conduce a hipocalcemia, conjuntamente con un aumento de la retención de fósforo y disminución de la vitamina D en los tejidos. Como mecanismo compensatorio, las células principales de las glándulas paratiroides sufren hiperplasia y producen mayor cantidad de paratohormona, dando como resultado el cuadro de hiperparatiroidismo secundario.

Si bien el tumor pardo es una lesión descrita mayormente en hiperparatiroidismo primario y puede ser la primera manifestación de esta enfermedad (32), el presente reporte contribuye con un caso adicional a los descritos en hiperparatiroidismo secundario como tumor óseo localizado y expansivo ubicado en la mandíbula. Los estudios tomográficos de la región maxilofacial contribuyeron a identificar mejor las características de las alteraciones óseas que ocurrían a nivel de los maxilares.

Finalmente, es importante enfatizar que el diagnóstico histopa- tológico del tumor pardo se realizó mediante un procedimiento simple de punción- aspiración, el cual podemos afirmar, en nuestro caso resultó ser un método confiable, fácil y libre de complicaciones.

\section{Referencias bibliográficas}

1. Clark OH, Wilkes W, Siperstein AE, Duh QY. Diagnosis and management of asymptomatic hyperparathyroidism: safety, efficacy, and deficiencies in our knowledge. J Bone Miner Res. 1991; 6 Suppl 2:S135-42.

2. National Institute of Health. Diagnosis and management of asymptomatic primary hyperparathyroidism. National Institute of Health Consensus Development Conference. Consens Statement. 1990; 8:29-31.

3. Triantafillidou K, Zouloumis L, Karakinaris G, Kalimeras E, Iordanidis F. Brown tumors of the jaws associated with primary or secondary hyperparathyroidism. A clinical study and review of the literature. Am J Otolaryngol. 2006; 27(4):281-6.

4. Yoshimoto K, Yamasaki R, Sakai H, Tezuka U, Takahashi M, Iizuka M, Sekiya T, Saito S. Ectopic production of parathyroid hormone by small cell lung cancer in a patient with hypercalcemia. J Clin Endocrinol Metab. 1989; 68(5):976-81.

5. Strewler GJ, Budayr AA, Bruce RJ, et al. Secretion of authentic parathyroid hormone by a malignant tumor. Clin Res. 1990; 38:462A.

6. Ruda JM, Hollenbeak CS, Stack BC Jr. A systematic review of the diagnosis and treatment of primary hyperparathyroidism from 1995 to 2003. Otolaryngol Head Neck Surg. 2005; 132(3):35972.

7. Billy HT, Rimkus DR, Hartzman S, Latimer RG. Technetium99m-sestamibi single agent localization versus high resolution ultrasonography for the preope- 
rative localization of parathyroid glands in patients with primary hyperparathyroidism. Am Surg. 1995; 61(10):882-8.

8. Boonen $\mathrm{S}$, Vanderschueren D, Pelemans W, Bouillon R. Primary hyperparathyroidism: diagnosis and management in the older individual. Eur J Endocrinol. 2004; 151(3):297-304.

9. Silverman S Jr, Ware WH, Gillooly C Jr. Dental aspects of hyperparathyroidism. Oral Surg Oral Med Oral Pathol. 1968; 26(2):184-9.

10.Drüeke T, Kubrusly M. Pathogenesis of secondary hyperparathyroidism of chronic renal failure. J Nephrol. 1994; 7:13947.

11. Martin KJ, Olgaard K, Coburn JW, Coen GM, Fukagawa M, Langman C, Malluche $\mathrm{HH}$, McCarthy JT, Massry SG, Mehls O, Salusky IB, Silver JM, Smogorzewski MT, Slatopolsky EM, McCann L; Bone Turnover Work Group. Diagnosis, assessment, and treatment of bone turnover abnormalities in renal osteodystrophy. Am J Kidney Dis. 2004; 43(3):558-65.

12. Okada H, Davies JE, Yamamoto H. Brown tumor of the maxilla in a patient with secondary hyperparathyroidism: a case study involving immunohistochemistry and electron microscopy. J Oral Maxillofac Surg. 2000; 58(2):233-8.

13. Aggunlu L, Akpek S, Coskun B. Leontiasis ossea in a patient with hyperparathyroidism secondary to chronic renal failure. Pediatr Radiol. 2004; 34(8):630-2.

14.Etemadi J, Mortazavi-Khosrowshahi M, Ardalan MR, Esmaili H, Javadrashid R, Shoja MM. Brown tumor of hyperparathyroidism masquerading as central giant cell granuloma in a renal transplant recipient: a case report. Transplant Proc. 2009; 41(7):2920-2.

15.Nieto J, Ruiz-Cuevas P, Escuder A, Regas J, Callis L. Tertiary hyperparathyroidism after renal transplantation. Pediatr Nephrol. 1997; 11(1):65-8.

16.Pitt SC, Sippel RS, Chen H. Secondary and tertiary hyperparathyroidism, state of the art surgical management. Surg Clin North Am. 2009; 89(5):122739.

17. Moe S, Drüeke T, Cunningham J, Goodman W, Martin K, Olgaard K, Ott S, Sprague S, Lameire N, Eknoyan G; Kidney Disease: Improving Global Outcomes (KDIGO). Definition, evaluation, and classification of renal osteodystrophy: a position statement from Kidney Disease: Improving Global Outcomes (KDIGO). Kidney Int. 2006; 69(11):1945-53.

18. Hruska K. Pathophysiology of renal osteodystrophy. Pediatr Nephrol. 2000; 14(7):636-40.

19.Kelly WH, Mirahmadi MK, Simon JH, Gorman JT. Radiographic changes of the jawbones in end stage renal disease. Oral Surg Oral Med Oral Pathol. 1980; 50(4):372-81.

20.Nathan AS, Traiger J, Berman SA. Secondary hyperparathyroidism as a cause of generalized enlargement of the maxilla and mandible. Report of a case. Oral Surg Oral Med Oral Pathol. 1966; 21(6):724-31.

21.Phelps KR, Bansal M, Twersky J. Jaw enlargement complicating secondary hyperparathyroidism in three hemodialysis patients. Clin Nephrol. 1994; 41(3):1739.

22. Michiwaki Y, Michi K, Yamaguchi A. Marked enlargement of the jaws in secondary hyperparathyroidism--a case report. Int J Oral Maxillofac Surg. 1996; 25(1):54-6.

23.Damm DD, Neville BW, McKenna S, Jones AC, Freedman PD, Anderson WR, Allen CM. Macrognathia of renal osteodystrophy in dialysis patients. Oral Surg Oral Med Oral Pathol Oral Radiol Endod. 1997; 83(4):489-
95.

24. Adornato MC, Mayne RW. Macrognathia of renal osteodystrophy in a dialysis patient. Report of a case. N Y State Dent J. 2000; 66(10):30-4.

25. Vigneswaran N. Oral and maxillofacial pathology case of the month. Renal-osteodystrophy induced macrognathia. Tex Dent J. 2001; 118(7):570-1, 582-3.

26. Asaumi J, Aiga H, Hisatomi M, Shigehara H, Kishi K. Advanced imaging in renal osteodystrophy of the oral and maxillofacial region. Dentomaxillofac Radiol. 2001; 30(1):59-62.

27. Rothstein T, Schneider R. Palatal swelling and lupus erythematosus. Macrognathia of renal osteodystrophy. Gen Dent. 2003; 51(1):78, 81.

28. Kalyvas D, Tosios KI, Leventis MD, Tsiklakis K, Angelopoulos AP. Localized jaw enlargement in renal osteodystrophy: report of a case and review of the literature. Oral Surg Oral Med Oral Pathol Oral Radiol Endod. 2004; 97(1):68-74.

29. Hata T, Irei I, Tanaka K, Nagatsuka H, Hosoda M. Macrognathia secondary to dialysis-related renal osteodystrophy treated successfully by parathyroidectomy. Int J Oral Maxillofac Surg. 2006; 35(4):378-82.

30.Lerman MA, Do C, Gunaratnam L, Kulkarni C, Tucker K, Woo SB. Localized mandibular enlargement in end-stage renal disease: two case reports and a review of the literature. Oral Surg Oral Med Oral Pathol Oral Radiol Endod. 2011; 113(3):38490.

31.Fordham CC 3rd, Williams TF. Brown tumor and secondary hyperparathyroidism. N Engl J Med. 1963; 269:129-31.

32.Suarez-Cunqueiro MM, Schoen R, Kersten A, Klisch J, Schmelzeisen R. Brown tumor of the mandible as first manifestation of atypical parathyroid adenoma. J Oral Maxillofac Surg. 2004; 62(8):1024-8. 